\title{
THE LACK OF CHEMICAL EQUILIBRIUM DOES NOT PRECLUDE THE USE OF CLASSICAL NUCLEATION THEORY IN CIRCUMSTELLAR OUTFLOWS
}

\author{
JOHN A. PAQUETTE \\ Astrochemistry Laboratory, Code 691, NASA's Goddard Space Flight Center, Greenbelt, MD 20771, USA \\ AND JOSEPH A. NUTH III \\ Solar System Exploration Division, Code 690, NASA's Goddard Space Flight Center, Greenbelt, MD 20771, USA
}

\begin{abstract}
Classical nucleation theory has been used in models of dust nucleation in circumstellar outflows around oxygen-rich asymptotic giant branch stars. One objection to the application of classical nucleation theory (CNT) to astrophysical systems of this sort is that an equilibrium distribution of clusters (assumed by $\mathrm{CNT}$ ) is unlikely to exist in such conditions due to a low collision rate of condensable species. A model of silicate grain nucleation and growth was modified to evaluate the effect of a nucleation flux orders of magnitude below the equilibrium value. The results show that a lack of chemical equilibrium has only a small effect on the ultimate grain distribution.
\end{abstract}

\section{Introduction}

Oxygen-rich AGB (asymptotic giant branch) stars are surrounded by a large and dense shell of cool gas and dust, the product of an outflow of gas from the star and the condensation of dust once that gas has expanded and cooled sufficiently in the process of traveling away from its parent. The condensation of silicate dust in circumstellar envelopes was first considered by Kamijo (1963). Silicon monoxide has been considered to be a likely candidate for dust nucleation (Nuth and Donn, 1982; Gail and Sedlmayr, 1986) because it is the 
most abundant reactive oxygen-bearing species in O-rich circumstellar shells, suggesting that it will be the first species to condense from the gas phase. $\mathrm{SiO}$ also has a very high bond energy such that it is stable even at high temperatures; $\mathrm{SiO}$ had been observed to condense at $1000 \mathrm{~K}$ in laboratory conditions. Although maser emission lines confirm the presence of $\mathrm{SiO}$ molecules close to such stars, and the observation of infrared emission or absorption bands which are associated with silicate grains (Habing, 1996, Danchi et al. 1994) provides evidence for the presence of $\mathrm{SiO}$ grains in their circumstellar shells, $\mathrm{SiO}$ was rejected by some researchers (e.g. Gail \& Sedlmayr 1998) as candidate for the formation of dust grains on the grounds that $\mathrm{SiO}$ nucleation could not occur in circumstellar outflows until the gas temperature had fallen below about $500 \mathrm{~K}$, while the formation of dust in the circumstellar shells of AGB stars is thought to occur around $1000 \mathrm{~K}$.

More recent measurements of the SiO vapor pressure (Nuth \& Ferguson 2006; Ferguson \& Nuth 2008) indicate that the vapor pressure curve commonly used (due to Schick, 1960) is significantly in error when applied to circumstellar outflows. The higher vapor pressures measured by Nuth and Ferguson together with a consideration of vibrational disequilibrium (Nuth \& Donn 1981; Nuth et al. 1985) suggest that silicate grains actually do nucleate in circumstellar outflows. Vibrational disequlibrium is posited to exist under low pressure, optically-thin conditions because collisional excitation and photon absorption cannot balance spontaneous emission processes for strong, energetic vibrational transitions in molecules such as SiO. A model of silicate grain nucleation and growth has been constructed, based on classical nucleation theory (Becker and Döring 1935), considering deviations from radiative equilibrium, and making use of the vapor pressure measurements of Ferguson \& Nuth (2008). This model calculates the effects of nucleation and growth using two different methods - a bin method which counts and tracks grains as they nucleate and grow, and a moment method that calculates the moments of the grain radius distribution using the technique of Sedlmayr and Dominic (1995). Quantities such as the total number density, the mean grain 
radius and the standard deviation of the grain radius are calculated from the moments. The two methods are independent, having only an outflow velocity and a temperature variation in common. The details of (and preliminary results from) this model are described in Paquette et al (2011). In this letter we consider one of the primary objections to the use of classical nucleation theory under such conditions - the probable lack of equilibrium among precondensation clusters, which contradicts one of the underlying assumptions of classical nucleation theory - and attempt to use the model of Paquette et al (2011) to gauge the seriousness of this objection.

\section{An Objection to the Use of Classical Nucleation Theory in an Astrophysical Environment}

Classical nucleation theory (hereafter CNT) was developed to deal with the transition from vapor to liquid in laboratory experiments under varying conditions of temperature and supersaturation. In CNT, condensation occurs by the formation and growth of small clusters. These precondensation clusters have positive free energy, and are thus unstable; energy considerations favor cluster shrinkage rather than cluster growth. In the systems for which CNT was developed a steady-state distribution of such small clusters is swiftly attained, ranging from on the order of a nanosecond for typical nucleation experiments (Abraham, 1974) to a few tenths of a second for the binary nucleation of atmospheric droplets (Schelling \& Reiss, 1981). At sufficient partial pressure of the condensing species, a critical cluster size is reached, which has maximal free energy. The addition of monomers to such clusters thus leads to a decrease in the free energy

of the clusters, encouraging the growth of larger grains. For SiO nucleation, CNT predicts that the nucleation flux of critical clusters, $\mathrm{J}$, is given by

$J=\left(\frac{2 \sigma}{\pi m_{S i O}}\right) n_{1}^{2} V_{S i O} \exp \left[\frac{-16 \pi \sigma^{3} V_{S i O}^{3}}{3(k T)^{3}(\ln S)^{2}}\right]$ 
where $\sigma$ is the surface free energy of $\mathrm{SiO}, m_{S i O}$ is the mass and $V_{S i O}$ the volume of an SiO molecule, $n_{l}$ is the number density of $\mathrm{SiO}$ monomers, $k$ is Boltzmann's constant, $T$ is the temperature, and $S$ the supersaturation of $\mathrm{SiO}$. The supersaturation is given in turn by ratio of $\mathrm{SiO}$ partial pressure (initially determined by the total pressure and the abundance of $\mathrm{Si}$, but decreased by condensation out of the gas phase once nucleation and growth begin) and the $\mathrm{SiO}$ equilibrium vapor pressure, which decreases as the gas cools. In the model described by Paquette et al. (2011) the temperature is assumed to decrease as $\mathrm{r}^{-1 / 2}$ (Kozasa \& Hasegawa 1984), an approximation that is valid for an optically thin shell with frequencyindependent opacity.

Donn \& Nuth (1985) argued that classical nucleation theory was not applicable to astrophysical systems like novae, protostellar nebulae, or circumstellar shells on theoretical grounds. While the initial distribution of precritical clusters is rapidly attained under laboratory conditions, Donn \& Nuth argued that this would not be the case in astrophysical systems because the density of monomers in astrophysical systems (on the order of $10^{-8}$ particles per cc) is in general, much lower than in laboratory conditions $\left(10^{14}\right.$ particles per cc or more). This leads to a low collision rate between monomers, and slows the progress to an equilibrium cluster distribution. If the time needed to achieve an equilibrium cluster distribution is not short compared to the time in which the gas density and temperature change significantly (due to expansion occurring as a consequence of the outflow), the assumptions underlying CNT are not met, and Equation 1 will not apply.

CNT has been used to describe silicate nucleation in circumstellar shells by numerous researchers (Donn et al, 1968; Draine \& Saltpeter 1977; Gail et al 1984; Gail \& Sedlmayr 1986; 1988; Sedlmayr \& Dominik 1995; Jeong et al. 2003; Nuth \& Ferguson, 2006). Still, since CNT was developed to deal with the 
transition from vapor to liquid under markedly different conditions than those pertaining in astrophysical environments, it is certainly reasonable to question its applicability in circumstellar shells. If equilibrium of the precondensation clusters is not attained, what are the consequences for the use of CNT? In other words would the lack of an equilibrium population lead to very different results than a model that uses the nucleation flux predicted by CNT (and thus implicitly assumes the existence of such an equilibrium population)?

\section{The Effect of a Deficit of Critical Clusters}

We used a simple method to estimate the degree of inaccuracy resulting from the use of CNT posed by a departure from an equilibrium cluster distribution. We assumed that the departure from equilibrium would lead to a deficit of critical clusters, as compared to the prediction of $\mathrm{CNT}$. We simulated this by reducing the nucleation flux by a factor $\beta$, i.e. the reduced nucleation flux would be given by $J^{\prime}=\beta J$, where $\mathrm{J}$ is as shown in Equation 1. So a small $\beta$ indicates a large departure from equilibrium (and a much smaller nucleation flux than CNT would predict given the same conditions), while $\beta=1$ would correspond to the prediction of CNT. We used the model described in Paquette et al. (2011) to gauge the effect of varying $\beta$ over a range of values.

Initially $\beta$ was set to $10^{-4}$ corresponding to a large comparative deficit of critical clusters. The effect of this on grain radius and nucleation flux is shown in Figure 1. The surface free energy, $\sigma$, for $\mathrm{SiO}$ is assumed to be $450 \mathrm{ergs} \mathrm{cm}^{2}$, the sticking coefficient for growth, $\alpha$, is assumed to be 0.01 , and the dilution factor of $\mathrm{W}=0.5$ indicates the degree of vibrational disequilibrium. The lower panel shows nucleation flux, $J$, from both the bin and moment methods for $\beta=1$ (open circles, thin line) and $\beta=10^{-4}$ (filled circles, thick line) plotted as a function of radial distance from the star. For both values of $\beta$ the bin and moment curves 
agree extremely well and essentially overlay each other. The upper panel shows the grain radius distributions after all $\mathrm{SiO}$ has condensed from the vapor phase. Again, the results of both the bin and moment methods are plotted for $\beta=1$ (open circles, thin line) and $\beta=10^{-4}$ (filled circles, thick line). Since the moment method provides the moments of the grain radius distribution rather than the distribution itself, a log-normal form has been assumed for the purposes of plotting the moment method data. The grain radius distributions from the bin and moment methods differ appreciably, but the mean grain radius from the two methods is almost the same - about $0.54 \mathrm{~nm}$. The grain distributions corresponding to $\beta=10^{-4}$ are characterized by a lower mean radius (around $0.46 \mathrm{~nm}$ ), just as one might expect. This inevitably means that the peak number density is higher for the $\beta=10^{-4}$ case, because the same mass of SiO went into grains in both simulations. But while the grain radius has decreased, it is interesting to note that the magnitude of this effect is far less than one might naively expect. A factor of 10,000 decrease in the nucleation flux leads to a corresponding decrease in the mean grain radius of only about $15 \%$. The key to understanding this lies in the lower panel of Figure 1. At the radius where nucleation begins, the nucleation flux for the small $\beta$ case is much less than for the $\beta=1$ case just as expected. However, the nucleation flux swiftly rises, peaking at about a quarter of a stellar radius further out than in the $\beta=1$ case. The height of the peak is similar to the peak in the $\beta=1$ case, so that a similar number of grains is produced, and the conditions at the location those grains are nucleated are only a little different than in the $\beta=1$ case, so the mean grain size ends up being similar as well.

Of course, the nucleation flux in the $\beta=10^{-4}$ case is only guaranteed to be 10,000 times lower than in the $\beta=1$ case if all other conditions are unchanged. Reducing $J$ by such a factor also affects the supersaturation, $\mathrm{S}$, of $\mathrm{SiO}$. Since $\mathrm{J}$ is 10,000 times smaller as nucleation begins, the number of nucleating grains is reduced by the same factor, and much less of the $\mathrm{SiO}$ is depleted from the gas. This leads to a 
greater $\mathrm{S}$ at greater distances from the star as compared with the $\beta=1$ case. Since Equation 1 depends on $\mathrm{S}$ through an exponential, the increased supersaturation greatly increases the nucleation flux. The amount of increase swiftly overwhelms the prefactor of $10^{-4}$ and causes the nucleation flux to attain values similar to those in the $\beta=1$ case, albeit at a slightly larger distance from the star. Strangely, it is the great sensitivity of the nucleation flux to $S$ that causes the insensitivity to $\beta$.

Figure 2 shows the effect of varying degrees of deficit in the nucleation flux as compared to the predictions of CNT. The mean grain radius from the bin and moment methods (open and filled circles, left axis) and the location of peak nucleation flux (triangles, right axis) are plotted for 6 values of $\beta$ ranging from $10^{-4}$ to unity. The solid and dashed lines represent power law fits to the mean grain radius from the bin method and moment method, respectively. The dotted line is a power law fit to the distance from the star at which nucleation flux peaked. The correlation coefficient is approximately 0.999 in each case. The data show a weak (about $15 \%$ over 4 orders of magnitude) monotonic increase for grain radius as a function of $\beta$ and a weak (about $5 \%$ over 4 orders of magnitude) monotonic decrease for the radius of the nucleation peak as a function of $\beta$. The trend suggests that even fairly radical decreases in nucleation flux have a relatively small effect on the final grain distribution, e.g. it appears that decreasing the nucleation flux by a factor of a million would lead to only a $20 \%$ decrease in mean grain radius.

The grains produced by the model are much smaller than sub-micron size typical in the interstellar medium. This is because this simple model does not take coagulation into account. The addition of coagulation to the model (which is planned as future work) will lead to larger grains.

\section{Summary and Conclusion}


One objection to the use of classical nucleation theory is astrophysical environments has been that a lack of chemical equilibrium (due to a low collision rate, in turn resulting from a low monomer number density) violates one of the assumptions underlying CNT. Model calculations in which the number of critical clusters was depressed far below that predicted by CNT show that the effect of such a depression on grain radius and the location of peak nucleation flux is anything but severe. Even large decreases in nucleation flux (relative to the prediction of CNT) affect the mean grain radius by $15 \%$ or less, and the location of peak nucleation by $5 \%$ or less. This result suggests that the use of nucleation theory in circumstellar outflows is not unreasonable. Even if there is a deficit of critical clusters, and even if that deficit is a large one, the resultant grains will be but little changed in size or number density.

J.A.P. acknowledges support from NASA's Astrophysics Data Program. J.A.N. acknowledges support from NASA's Cosmochemistry Program.

\section{References}

Abraham, F.F., 1974, Homogeneous Nucleation Theory (New York: Academic Press)

Becker, R., \& Doring, W., Annaelen der Physik, 1935, 24, 719

Danchi, W.C., Bester, M., Degiacomi, C.G., Greenhill, L.J., \& Townes, C.H., 1994, AJ, 107, 1469

Donn, B.D., \& Nuth, J. A., ApJ, 1985, 288, 187

Donn, B., Wickramasinghe, N.G., Hudson, J., \& Stecher, T., 1968, ApJ, 153, 451

Draine B.T. \& Salpeter, E.E., 1977, Journal of Chemical Physics, 77, 2230

Ferguson, F.T., \& Nuth J.A., 2008, Journal of Chemical Engineering Data, 53, 2824

Gail, H.P., Keller, R., \& Sedlmayr, E., 1984, A\&A, 133, 320

Gail, H.P. \& Sedlmayr, E., 1986, A\&A, 166, 225 
Gail, H. and E. Sedlmayr, 1998, Dust Formation in M Stars, in Chemistry and Physics of Molecules and Grains in Space, Faraday Discussions, 109, 303

Habing, H.J., 1996, A\&AR, 7, 97

Jeong, K.S., Winters, J.M., Le Bertre, T., \& Sedlmayr, E., 2003, A\&A, 407, 191

Kamijo, F., 1963, PASJ, 15, 440

Kozasa, T. \& Hasegawa, H., 1987, Progress of Theoretical Physics, 77, 1402

Nuth, J.A., \& Donn, B., 1981, ApJ, 247, 925

Nuth, J.A. \& Donn, B., 1982, Journal of Chemical Physics, 77, 2639

Nuth, J.A. \& Ferguson, F. T., 2006, ApJ, 649, 1178

Nuth, J.A., Wiant, M., \& Allen, J., 1985, ApJ, 293, 463

Paquette, J.A., Ferguson, F. T., \& Nuth, J.A., 2011, ApJ, 732, 62

Schick, H. L., Chemistry Reviews, 1960, 60, 331

Sedlmayr, E., \& C. Dominik, C., 1995, Space Science Reviews, 73, 211

Schelling, F.J. \& Reiss, H., 1981, Journal of Chemical Physics, 74, 3527 

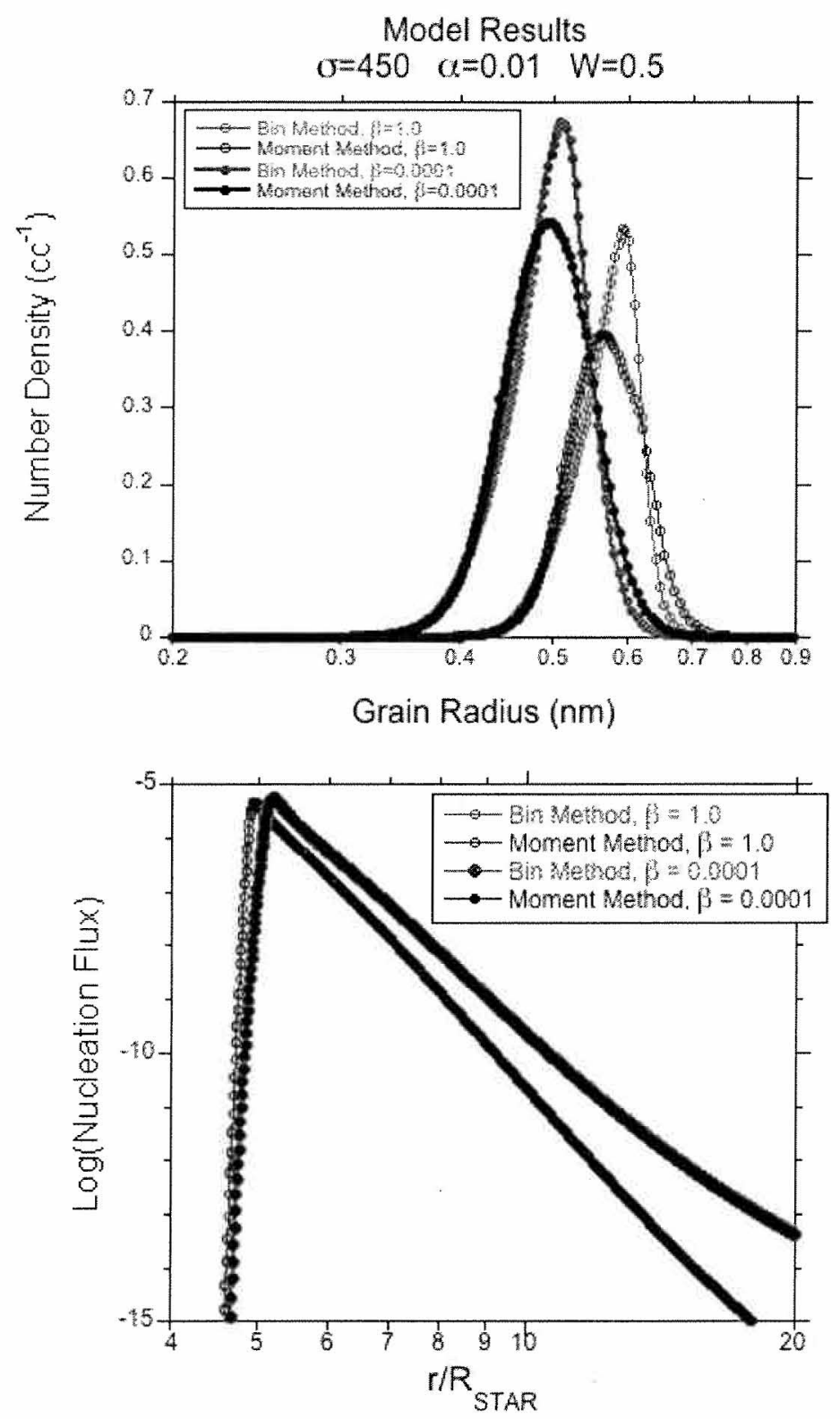

Fig. 1 The effect of changing the flux factor $(\beta)$ on nucleation flux $(J)$ and the grain radius distribution. The upper panel shows nucleation flux for the bin and moment methods for $\beta=1$ and $\beta=0.0001$. For each $\beta$ value, the bin and moment curves are virtually identical, but the smaller $\beta$ nucleates further from the star. The lower panel shows the grain radius distributions (again from the bin and moment methods) for $\beta=1$ and $\beta=0.0001$. Decreasing $\beta$ leads to smaller grains (and a larger number of grains, as the mass contained in grains is the same), but the change is not extreme despite a large variation in $\beta$. 


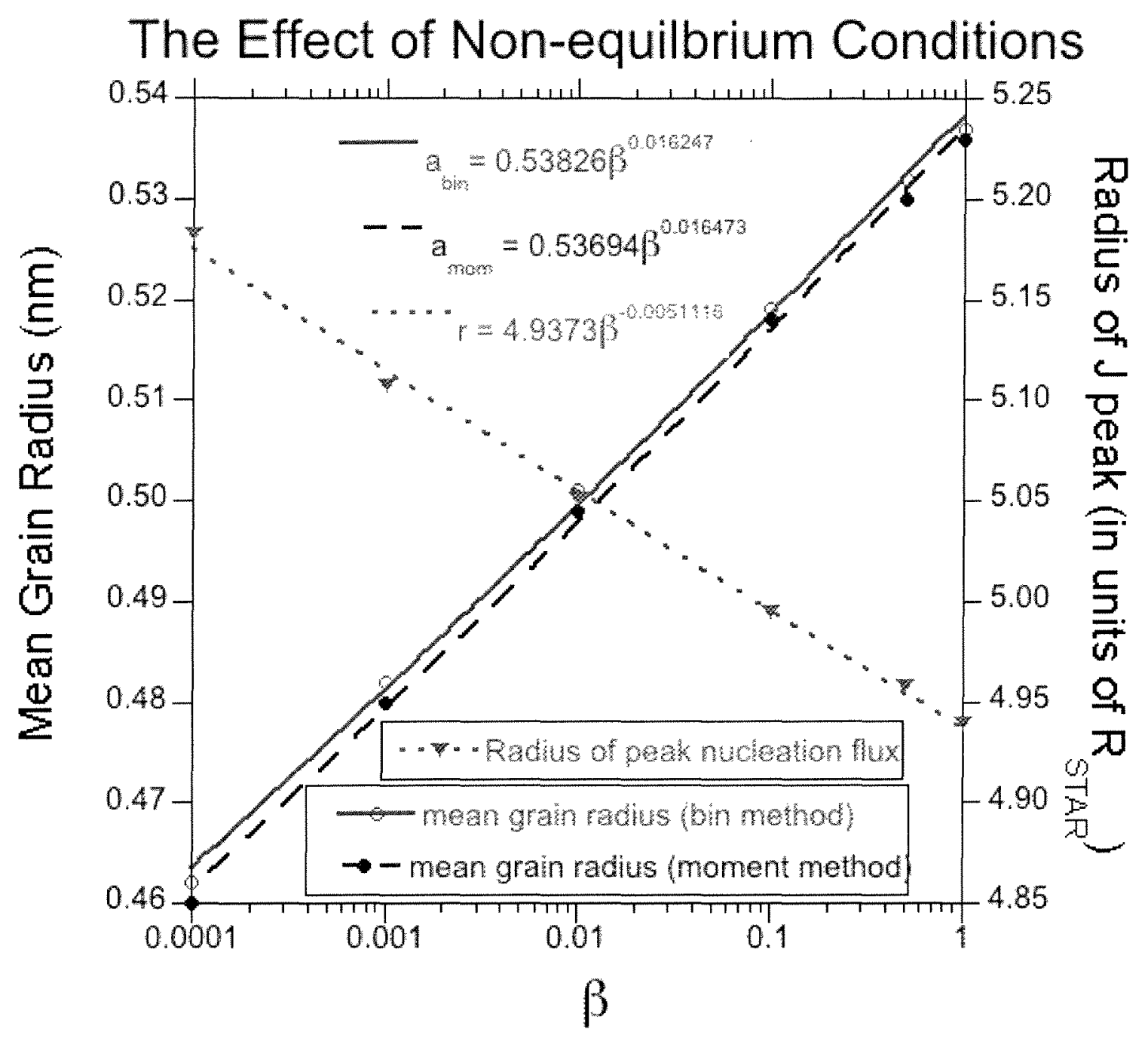

Fig. 2 The effect of varying the flux factor, $\beta$, on nucleation flux and grain radius. The circles are the mean grain radius from the bin and moment methods as a function of $\beta$. The solid (dashed) line is a power law fit to the bin method (moment method) data. For either method, a factor of 10,000 in $\beta$ changes the mean grain radius by less than $15 \%$. The triangles are the radius of peak nucleation (in units of the stellar radius) as a function of $\beta$. The dotted line is a power law fit to the peak radius data. The nucleation peak moves inward as $\beta$ increases. The correlation coefficient for all 3 fits is 0.999 . 